PROCEEDINGS OF THE AMERICAN MATHEMATICAL SOCIETY

Volume 124, Number 9, September 1996

\title{
WEAK-TYPE ENDPOINT BOUNDS FOR RIESZ MEANS
}

TERENCE TAO

(Communicated by Christopher D. Sogge)

\begin{abstract}
We use $L^{2}$ restriction theory to prove optimal weak-type bounds of Bochner-Riesz multipliers and Riesz means of elliptic pseudo-differential operators on compact manifolds, for $p \leq 2(n+1) /(n+3)$.
\end{abstract}

\section{INTRODUCTION}

Consider the Bochner-Riesz multipliers ${ }^{1} S^{\delta}=\left(1-|D|^{2}\right)_{+}^{\delta}$ in dimension $n \geq 2$. It is conjectured that, for $1 \leq p \leq 2 n /(n+1)$, these operators are of strong-type $(p, p)$ precisely when $\delta>\delta(p)=n(1 / p-1 / 2)-1 / 2$. Progress on this conjecture can be found in [7], [2], [1]; it remains open for $n>2$ and $p$ close to $2 n /(n+1)$.

A natural strengthening of the above conjecture is the claim that $S^{\delta(p)}$ is of weak-type $(p, p)$ whenever $1 \leq p<2 n /(n+1)$. (The necessity of the condition on $p$ was shown in [8]). In the special $n=2$ case the full conjecture has been proven by Seeger [12]. In general dimension the conjecture was proven by Christ [5], [4] for any $p<p_{0}$, where $p_{0}$ is such that there is a $\left(p_{0}, 2\right)$ restriction theorem for the sphere in $\mathbb{R}^{n}$. Thus, by the well-known restriction theorem of Tomas and Stein, the conjecture is true for $p<2(n+1) /(n+3)$.

Our first result is that the conjecture also holds at the endpoint $p=2(n+1) /$ $(n+3)$.

Theorem 1.1. Suppose there is a $(p, 2)$ restriction theorem for the sphere in $\mathbb{R}^{n}$. Then the operator $S^{\delta(p)}$ is of weak-type $(p, p)$.

In fact, under the restriction theorem hypothesis we have the stronger estimate

$$
\int_{M_{p} f(x) \leq \alpha}\left|S^{\delta(p)} f(x)\right|^{2} d x \leq C \alpha^{2-p}\|f\|_{p}^{p} ;
$$

here $M_{p} f=M\left(|f|^{p}\right)^{1 / p}$ and $M$ is the Hardy-Littlewood maximal function.

We also show the analogous result for Riesz means on compact manifolds. More precisely, suppose $P$ is a first-order self-adjoint elliptic pseudo-differential operator with nonnegative eigenvalues on a smooth compact manifold $M$. We say that there is a (discrete) $(p, 2)$ restriction theorem for $P$ if $\left\|\chi_{[k, k+1]}(P)\right\|_{(p, 2)} \leq C(1+k)^{\delta(p)}$ for all $k>0$, and consider the Riesz means $S_{R}^{\delta}=\left(1-\frac{P}{R}\right)_{+}^{\delta}$, defined for $R, \delta>0$. In Sogge [14] it is shown that, if there is a $(p, 2)$ restriction theorem for $P$, then $S_{R}^{\delta}$ is of strong type $(p, p)$ uniformly in $R$, for $\delta>\delta(p)$. Also, it was shown by Christ

Received by the editors March 14, 1995.

1991 Mathematics Subject Classification. Primary 42B15.

${ }^{1}$ Our notation will be explained more fully in subsequent sections.

(C)1996 American Mathematical Society 
and Sogge [6] that $S_{R}^{\delta(1)}$ is weak $(1,1)$ uniformly in $R$ for any $P$. Combining ideas from these results with the methods of [4], it was shown by Seeger [11] that $S^{\delta(p)}$ was weak $(p, p)$ uniformly in $R$ for $p<p_{0}$, if one has a $\left(p_{0}, 2\right)$ restriction theorem for $P$.

By modifying the proof of Theorem 1.1, we have an endpoint version of the result in [11].

Theorem 1.2. Suppose there is a $(p, 2)$ restriction theorem for $P$. Then the operators $S_{R}^{\delta(p)}$ are of weak-type $(p, p)$ uniformly in $R$.

As in the Euclidean case, we have the stronger estimate

$$
\int_{M_{p} f(x) \leq \alpha}\left|S_{R}^{\delta(p)} f(x)\right|^{2} d x \leq C \alpha^{2-p}\|f\|_{p}^{p}
$$

holding uniformly in $R$. (The bound $C$ depends on the balls used to define $M_{p}$ ).

It is known that the $(1,2)$ restriction theorem holds for any $P$. Furthermore, under an additional curvature assumption, one has a $(p, 2)$ restriction theorem for all $1 \leq p \leq 2(n+1) /(n+3)$. See [13],[15],[16]. Combining these results with $(2)$ and interpolating with the trivial case $\delta \geq 0, p=2$, one has a corollary:

Corollary 1.3. Suppose $P$ is such that the co-spheres $\left\{\xi \in T_{x}^{*} M \backslash 0: p(x, \xi)=1\right\}$ have everywhere non-vanishing Gaussian curvature for each $x \in M$. Then the operators $S_{R}^{\delta}$ are of weak-type $(p, p)$ uniformly in $R$ when $1 \leq p \leq 2$ and $\delta \geq$ $\max \left(\delta(p), \frac{n-1}{2}\left(\frac{1}{p}-\frac{1}{2}\right)\right)$.

The method of proof of Theorems 1.1 and 1.2 is a modification of that used by Christ [4], which involved $L^{2}$ Calderón-Zygmund techniques (as used in [7]), a dyadic decomposition of the Bochner-Riesz multiplier with special properties, and the Cauchy-Schwarz inequality. Our main innovations are the replacement of the dyadic decomposition with a decomposition into two terms (which depend on the level of resolution), and a greater reliance on a certain "locality" principle. In the Euclidean case the principle is the trivial observation that if a multiplier $m$ has its inverse Fourier transform supported on a set of width $R$, then the operator $m(D)$ is local at the scale ${ }^{2}$ of $R$. On compact manifolds the corresponding principle is that if a function $m$ on $\mathbb{R}$ has its Fourier transform supported on an interval of width $R$, then the operator $m(P)$ is - apart from an error with good decay-local at the scale of $R$.

\section{Proof of Theorem 1.1}

If $I$ is a cube, we denote its side-length by $l(I)$, and for any $c>1, c I$ will denote the cube with the same center but with side-length $\operatorname{cl}(I)$. If $E$ is a set, $|E|$ will denote the Lebesgue measure of $E$. If $m$ is a function on $\mathbb{R}^{n}$, we will denote by $m(D)$ the operator corresponding to the multiplier $m$, thus $(\widehat{m(D) f})(\xi)=m(\xi) \hat{f}(\xi)$. We use the letter $C$ to stand for various (large) constants.

Fix $n \geq 2$, and assume that $1 \leq p<2$ is such that a $(p, 2)$ restriction theorem holds for the sphere. Write $S^{\delta}=m^{\delta}(D)$, where $m^{\delta}(\xi)=\left(1-|\xi|^{2}\right)_{+}^{\delta}$ and $\delta=\delta(p)=$ $n(1 / p-1 / 2)-1 / 2$. We have to show that $m^{\delta}(D)$ is of weak-type $(p, p)$. Since $M_{p}$ is

\footnotetext{
${ }^{2} \mathrm{By}$ this we mean that the kernel of the operator is supported in a $C R$-neighbourhood of the diagonal of $\mathbb{R}^{n} \times \mathbb{R}^{n}$.
} 
of weak-type $(p, p)$, it suffices by Tchebyshev's inequality to prove (1). By linearity we may assume that $\alpha=C^{-1}$ for some large $C$ to be determined later.

Fix $f$, and apply the dyadic Calderón-Zygmund decomposition at height $C$ to $|f|^{p}$. This allows us to write $f=g+\sum_{I} b_{I}$, where $\|g\|_{\infty} \leq C$, the $b_{I}$ are supported on disjoint dyadic cubes $I$ and satisfy $\left\|b_{I}\right\|_{p} \sim|I|^{1 / p}$, and the $I$ are such that $\sum_{I}|I| \leq C\|f\|_{p}^{p}$. Note that $M_{p} f(x)>C^{-1}$ whenever $x \in \bigcup_{I} C I$.

Since $m^{\delta}$ is bounded, the contribution of $g$ to (1) is acceptable. In fact, as $m^{\delta}$ is also compactly supported, we may similarly dispose of the contribution of the small cubes. ${ }^{3}$ Specifically, let $\tilde{g}$ be $g+\sum_{l(I) \leq 1} b_{I}$ and choose a Gaussian $P$ that majorizes $m^{\delta}$. Then the contribution of $\tilde{g}$ is majorized by $\|P(D) \tilde{g}\|_{2}^{2}$. But the kernel of $P(D)$ is positive, integrable, and radial decreasing, so we have $\|P(D) \tilde{g}\|_{p} \leq C\|\tilde{g}\|_{p}$ and $\|P(D) \tilde{g}\|_{\infty} \leq C$. Thus $\|P(D) \tilde{g}\|_{2}^{2} \leq C\|\tilde{g}\|_{p}^{p} \leq C\|f\|_{p}^{p}$, which is acceptable.

Define $b_{i}$ to be $\sum_{l(I)=2^{i}} b_{I}$ for each $i>0$. From the above reductions, it suffices to show that

$$
\int_{\mathbb{R}^{n}-\cup_{I} C I}\left|\sum_{i=1}^{\infty} m^{\delta}(D) b_{i}(x)\right|^{2} d x \leq C \sum_{I}|I|
$$

to finish the proof. To estimate this expression we make a decomposition of the multiplier $m^{\delta}$ for each integer $i>0$, whose properties are summarized in the following lemma:

Lemma 2.1. For each $i>0$, there exists a decomposition $m^{\delta}=m_{i}+\eta_{i} n_{i}$ such that:

(i) The inverse Fourier transforms of $m_{i}$ and $n_{i}$ are supported in the ball of radius $2^{i}$ around the origin.

(ii) For all $\xi \in \mathbb{R}^{n}$, we have $\sum_{i=1}^{\infty}\left|\eta_{i}(\xi)\right|^{2} \leq C$.

(iii) For a certain large $N$, we have $\left|n_{i}(\xi)\right| \leq C 2^{-\delta i}\left(1+2^{i}|1-| \xi||\right)^{-N}$.

Before we prove this rather technical lemma, let us see how it implies (3). Write $m^{\delta}(D) b_{i}$ as $m_{i}(D) b_{i}+\eta_{i}(D) n_{i}(D) b_{i}$. By (i), the operator $m_{i}(D)$ is local at the scale of $2^{i}$, so $m_{i}(D) b_{i}$ is supported on $\bigcup_{l(I)=2^{i}} C I$ and makes no contribution to (3). Thus it suffices to prove $\left\|\sum_{i=1}^{\infty} \eta_{i}(D) n_{i}(D) b_{i}\right\|_{2}^{2} \leq C \sum_{I}|I|$. However, from (ii), Plancherel's formula, and the Cauchy-Schwarz inequality we see that this expression is bounded by $C \sum_{i=1}^{\infty}\left\|n_{i}(D) b_{i}\right\|_{2}^{2}$. So it will suffice to show that

$$
\left\|n_{i}(D) b_{i}\right\|_{2}^{2}=\left\|\sum_{l(I)=2^{i}} n_{i}(D) b_{I}\right\|_{2}^{2} \leq C \sum_{l(I)=2^{i}}|I|
$$

for each $i>0$. However, by (i) the operator $n_{i}(D)$ is local at the scale of $2^{i}$, so the individual $n_{i}(D) b_{I}$ have almost disjoint support. Thus we only need prove the above estimate for a single cube. But by Plancherel's formula, (iii), a change to

\footnotetext{
${ }^{3}$ This can be thought of as a dual to the more well-known principle that, if the kernel has compact support, then the large cubes can be safely discarded.
} 
polar co-ordinates, and the restriction theorem, we have

$$
\begin{aligned}
\left\|n_{i}(D) b_{I}\right\|_{2}^{2} & =\int_{\mathbb{R}^{n}}\left|n_{i}(\xi)\right|^{2}\left|\hat{b}_{I}(\xi)\right|^{2} d \xi \\
& \leq C \int_{0}^{\infty} 2^{-2 \delta(p) i}\left(1+2^{i}|1-r|\right)^{-2 N} \int_{S}\left|\hat{b}_{I}(r \theta)\right|^{2} d \theta r^{n-1} d r \\
& \leq C 2^{-2 \delta(p) i} \int_{0}^{\infty}\left(1+2^{i}|1-r|\right)^{-2 N} r^{-2 n / p^{\prime}}\left\|b_{I}\right\|_{p}^{2} r^{n-1} d r \\
& \leq C 2^{-2 \delta(p) i} 2^{-i}|I|^{2 / p} \\
& =C|I|,
\end{aligned}
$$

as desired, if $N$ is chosen to be sufficiently large.

It remains only to prove Lemma 2.1. Following [4], we start by choosing a smooth radial function $\phi$ supported on the unit ball and equal to 1 on the half-unit ball, such that $\int_{\mathbb{R}^{n}} \hat{\phi}(\xi)\left\langle\xi, e_{1}\right\rangle_{+}^{\delta} d \xi=0$ for some unit vector $e_{1}$. Write $\phi_{2^{i}}=\phi\left(2^{-i} \cdot\right)$, and set $m_{i}$ equal to $m^{\delta} * \hat{\phi}_{2^{i}}$. Thus $m_{i}$ obeys (i). Furthermore, since $\hat{\phi}_{2^{i}}$ is an approximation to the identity of "thickness" $2^{-i}$, one has by repeated integration by parts that

$$
\left|m^{\delta}(\xi)-m_{i}(\xi)\right| \leq C_{N} 2^{-\delta i}\left(2^{i}|1-| \xi||\right)^{-N}
$$

for $|1-| \xi||>2^{-i}$ and all $N>0$. When $|1-| \xi|| \leq 2^{-i}$, we claim that the additional estimate

$$
\left|m^{\delta}(\xi)-m_{i}(\xi)\right| \leq C 2^{-\delta i}\left(2^{-i}+2^{i}|1-| \xi||\right)^{\varepsilon}
$$

holds, where $\varepsilon=\min (\delta, 1)>0$. Since $(5)$ holds for $m^{\delta}(\xi)$, it suffices to prove the corresponding estimate for $m_{i}(\xi)$. However, by differentiating under the integral sign one can see that the gradient of $m_{i}(\xi)$ is $O\left(2^{i} 2^{-\delta i}\right)$ in the region of interest, so it suffices to prove (5) for $\xi$ on the unit sphere. Since $m_{i}$ is radial, it suffices to prove it for $\xi=e_{1}$. But this follows by approximating $m^{\delta}\left(e_{1}-\xi\right)$ by $2^{\delta}\left\langle\xi, e_{1}\right\rangle_{+}^{\delta}$ and using the assumed moment condition for $\hat{\phi}$. that

The next step will be to construct a positive function $n_{i}$ that obeys (i) and such

$$
C^{-1} 2^{-\delta i}\left(1+2^{i}|1-| \xi||\right)^{-M} \leq n_{i}(\xi) \leq C 2^{-\delta i}\left(1+2^{i}|1-| \xi||\right)^{-N}
$$

for some large $M, N$, which we reserve the right to choose later. Once we do this, it can easily be seen from (4), (5), and the above estimates that the function $\eta_{i}=\left(m^{\delta}-m_{i}\right) / n_{i}$ satisfies (ii), and so all the required properties will hold.

We begin by choosing some large even integer $N$ and considering the onedimensional function $\psi(\xi)=\left(\frac{\xi-\sin \xi}{\xi^{3}}\right)^{N / 2}$. By taking the tensor product of $n$ copies of $\psi$, averaging over the orthogonal group and then rescaling, we can construct a function $\psi_{n}$ on $\mathbb{R}^{n}$ which is positive and comparable to $(1+|\xi|)^{-N-n+1}$, and whose inverse Fourier transform is supported on the unit ball. It is then a routine matter to check that the function $n_{i}=2^{-\delta i} 2^{(n-1) i} \psi_{n}\left(2^{i} \cdot\right) * d \theta$ satisfies all the required properties, with $M=N+n-1$; here $d \theta$ is surface measure on the unit sphere. This completes the proof of Lemma 2.1, and the theorem follows.

Remarks 2.2. 1. The method of proof of Theorem 1.1, in particular the construction of $m_{i}$ and the use of the Cauchy-Schwarz inequality, is essentially due to Christ [4]. 
However, a slightly different decomposition is used in [4]; in our notation, it would be $m^{\delta}=m_{i}+\sum_{s=0}^{\infty}\left(m_{i+s+1}-m_{i+s}\right)$, where the individual $m_{i+s+1}-m_{i+s}$ play much the same role in [4] as the function $m^{\delta}-m_{i}=\eta_{i} n_{i}$ does here.

2. This method can provide alternate proofs of the weak $(1,1)$ type of various "rough" convolution operators. For example, the weak boundedness of $S^{(n-1) / 2}$ follows immediately from Theorem 1.1 , as the $(1,2)$ restriction theorem is trivial. Another example is the one-dimensional operator $f \mapsto f * e^{i \pi x^{2}} / x$. The multiplier, which is roughly $e^{-i \pi \xi^{2}} / \xi$, can be decomposed for each $i>0$ as $m_{i}+\eta_{i} n_{i}$, where (i) [for $m_{i}$ only] and (ii) of Lemma 2.1 holds and $n_{i}(\xi)=2^{-i}\left(1+2^{-i}|\xi|\right)^{-1+\varepsilon}$. We omit the details.

3. By interpolation, (1) will hold with $\delta(p)$ replaced by $\delta$, for $1 \leq p \leq 2$ and $\delta \geq \max \left(\delta(p), \frac{n-1}{2}\left(\frac{1}{p}-\frac{1}{2}\right)\right)$. Simple calculations involving bump functions on small balls or on thin tubes show that this region is best possible. In the interior of this region (1) also follows from the weighted inequality

$$
\int_{\mathbb{R}^{n}}\left|S^{\delta} g(x)\right|^{2} \psi(x) d x \leq C \int_{\mathbb{R}^{n}}|g|^{2} M_{r} \psi(x) d x
$$

proven by Christ [3]; here $r=p /(2-p)$ and $\psi$ is any positive function. Indeed, the adjoint of (6) is $\int_{\mathbb{R}^{n}} \frac{\left|S^{\delta} f(x)\right|^{2}}{M_{r} \psi(x)} d x \leq C \int_{\mathbb{R}^{n}} \frac{|f|^{2}}{\psi(x)} d x$, which yields (1) after substituting $\psi=|f|^{2-p}$ and using Tchebyshev's inequality. Unfortunately (6) fails for endpoint values of $\delta$, as the bump function examples will show.

\section{INTERMEZZO: A LOCALITY PRINCIPLE FOR FUNCTIONS OF ELLIPTIC PSEUDO-DIFFERENTIAL OPERATORS}

Let $M$ be a smooth compact connected manifold without boundary of dimension $n>1$, endowed with a smooth positive measure $d x$. It will be convenient to fix a smooth global Riemannian metric $d(x, y)$ on $M$.

Let $P=P(x, D)$ be a fixed first-order elliptic self-adjoint pseudo-differential operator on $M$. By elliptic we mean that the principal symbol

$$
p(x, \xi)=\lim _{\lambda \rightarrow \infty} \lambda^{-1} P(x, \lambda \xi)
$$

of $P$ exists and is positive for non-zero elements $\xi$ of the cotangent space $T_{x}^{*} M$.

It can be shown that the spectrum of $P$ is discrete. In particular, there exists a sequence of eigenvalues $\lambda_{j}$ tending to infinity and a sequence of mutually orthogonal rank one projections $e_{j}: L^{2} \rightarrow L^{2}$, such that $I=\sum_{j=1}^{\infty} e_{j}$ and $P=\sum_{j=1}^{\infty} \lambda_{j} e_{j}$ (on smooth functions at least). Since the eigenvectors of $P$ must belong to every Sobolev space, each projection $e_{j}$ must be smoothing, i.e. have a kernel that is in $C^{\infty}(M \times M)$. We will assume without loss of generality that the $\lambda_{j}$ are nonnegative.

We impose the obvious functional calculus for $P$, defining

$$
m(P) f=\sum_{j=1}^{\infty} m\left(\lambda_{j}\right) e_{j}(f)
$$

for tempered $m$ and smooth $f$. We will also use $m(P)$ to denote the (distributional) kernel of the above operator; thus $m(P) f(x)=\int_{M} m(P)(x, y) f(y) d y$. The main purpose of this section is to relate the "locality" (i.e. decay) properties of $m(P)(x, y)$ to those of $\hat{m}$. 
Proposition 3.1. Suppose $m$ is a function of $\mathbb{R}$ such that $\hat{m}$ is supported on $[-\tau, \tau]$ for some sufficiently small $\tau$. Then

(i) One has $|m(P)(x, y)| \leq C\|\hat{m}\|_{1} \tau d(x, y)^{-n-1}$ whenever $d(x, y)>C \tau$.

(ii) If in addition $m(\tau \cdot)$ is in $S^{-\infty}$, then $|m(P)(x, y)| \leq C \tau^{-n}\left(1+\frac{d(x, y)}{\tau}\right)^{-n-1}$ for all $x, y$.

Part (i) is a slightly stronger version of a result proved in [6]. The key idea is to exploit the fact that $e^{i t P}$ is local when $t=0$. Further improvements in the decay estimates (including asymptotics), as well as the relaxation of various hypotheses, are possible using variants of these ideas, together with some ideas of [11]. We will not pursue these matters here.

Proof. We first prove (i). By convexity we may assume that $m(P)$ is of the form $e^{i t P}$. But $e^{i t P}=I+\int_{0}^{t} \frac{d}{d s} e^{i s P} d s$, so it suffices to show that $\left|\frac{d}{d t} e^{i t P}(x, y)\right| \leq$ $C d(x, y)^{-n-1}$ when $d(x, y) / t$ is large.

To do this we shall apply a well-known approximation of $e^{i t P}$ by a Fourier integral operator. Specifically, if $\epsilon>0$ is sufficiently small, the (distributional) kernel of $e^{i t P}$ can be written for $|t| \leq \epsilon$ in the form

$$
e^{i t P}(x, y)=\eta_{\epsilon}(x, y) \int_{T_{y}^{*} M} e^{i \phi(x, y, \xi)} e^{i t p(y, \xi)} q(t, x, y, \xi) d \xi+E_{\epsilon}(t, x, y),
$$

where $\eta_{\epsilon}(x, y)$ is supported on a $C \epsilon$-neighbourhood of the diagonal of $M \times M$, $\phi(x, y, \xi)$ is a smooth real function such that $\left|\nabla_{\xi} \phi\right| \geq C^{-1} d(x, y)$ and $\left|\nabla_{\xi}^{k} \phi\right| \leq$ $C_{k} d(x, y)|\xi|^{1-k}$ for small $d(x, y)$ and $k \geq 1, q(t, x, y, \xi)$ is an $S^{0}$ symbol in $\xi$, and $E_{\epsilon}(t, x, y)$ is a $C^{\infty}$ error. One obtains (7) from a construction, originally due to Hörmander, of a local parametrix for the Cauchy problem for $P$. Details can be found in [10], [16].

If $d(x, y) / t$ is sufficiently large and $\epsilon$ is sufficiently small, then the phase $\Psi(t, x, y, \xi)=\phi(x, y, \xi)+t p(y, \xi)$ will satisfy the same sort of bounds as $\phi$ on the support of $\eta_{\epsilon}$. If we then differentiate (7), we obtain

$$
\frac{d}{d t} e^{i t P}(x, y)=\eta_{\epsilon}(x, y) \int e^{i \Psi}\left[\frac{d}{d t} q(t, x, y, \xi)+p(y, \xi) q(t, x, y, \xi)\right] d \xi+\frac{d}{d t} E_{\epsilon}(t, x, y) .
$$

The term $\frac{d}{d t} E_{\epsilon}(t, x, y)$ is $O(1)$ and can be discarded. Since the integrand is $O(1+|\xi|)$, the integral on the region $\left\{|\xi| \leq d(x, y)^{-1}\right\}$ is $O\left(d(x, y)^{-n-1}\right)$ and can also be discarded. Outside of this region, the term in brackets is - after a suitable rescaling by $d(x, y)$ - a symbol of order 1 , and so this part of the integral is also $O\left(d(x, y)^{-n-1}\right)$, by the standard estimates on non-stationary oscillatory integrals. Thus one has the desired bound on $\frac{d}{d t} e^{i t P}(x, y)$, which proves (i).

To show (ii), it suffices by (i) to prove $|m(P)(x, y)| \leq C \tau^{-n}$. From (7) we have

$$
m(P)(x, y)=\eta_{\epsilon}(x, y) \int e^{i \phi(x, y, \xi)}\left(\int e^{i t p(y, \xi)} q(t, x, y, \xi) \hat{m}(t) d t\right) d \xi+O(1) .
$$

But by the standard manipulations on symbols and the fact that $p(y, \xi) \sim|\xi|$, the inner integral will be $O_{N}(1+\tau|\xi|)^{-N}$ for all $N>0$. Taking $N>n$ thus gives the result.

We now show that operators with the decay of 3.1(i) are effectively local in a specialized $L^{p}$ sense. 
Proposition 3.2. Let $\{I\}$ be a collection of disjoint, sufficiently small "cubes" in $M$. Suppose that for each $I$ there is a function $b_{I}$ supported on $I$ and a function $m_{I}$ whose Fourier transform is supported on $[-l(I), l(I)]$ such that $\left\|b_{I}\right\|_{1} \leq C|I|$ and $\left\|\hat{m}_{I}\right\|_{1} \leq C$. Then, for $1 \leq p<\infty$, one has

$$
\left\|\sum_{I}\left(m_{I}(P) b_{I}\right) \chi_{C I^{c}}\right\|_{p}^{p} \leq C_{p} \sum_{I}|I| .
$$

In particular, if the $C I$ are almost disjoint, then

$$
\left\|\sum_{I} m_{I}(P) b_{I}\right\|_{p}^{p} \leq C_{p}\left(\sum_{I}\left\|m_{I}(P) b_{I}\right\|_{p}^{p}+\sum_{I}|I|\right) .
$$

Proof. It suffices to prove the first inequality. By Proposition 3.1(i) and elementary estimates, we see that $\left|m_{I}(P) b_{I}(x) \chi_{C I^{c}}(x)\right| \leq C\left(M \chi_{I}(x)\right)^{q}$, where $q=1+1 / n>1$. Thus

$$
\begin{aligned}
\left\|\sum_{I}\left(m_{I}(P) b_{I}\right) \chi_{C I^{c}}\right\|_{p}^{p} & \leq C\left\|\left(\sum_{I}\left(M \chi_{I}\right)^{q}\right)^{1 / q}\right\|_{p q}^{p q} \\
& \leq C_{p}\left\|\left(\sum_{I}\left(\chi_{I}\right)^{q}\right)^{1 / q}\right\|_{p q}^{p q}=C_{p} \sum_{I}|I|
\end{aligned}
$$

as desired, where we have used the vector-valued maximal theorem of Fefferman and Stein [9]. (It is a routine matter to adapt the theorem in [9] to general manifolds.)

\section{Proof of Theorem 1.2}

Let $1 \leq p<2$ be such that there is a $(p, 2)$ restriction theorem for $P$. We wish to show that $S_{R}^{\delta}$ is of weak type $(p, p)$ uniformly in $R$, where $\delta=\delta(p)$. Since the proof is almost identical to that of Theorem 1.1 except for a rescaling and some minor technical complications, we give only a brief sketch.

We first note that, since each projection operator is smoothing, the claim is clearly true when $R$ is bounded. Thus we may assume that $R>C$ for some large $C$ to be determined later.

Secondly, since the eigenvalues of $P$ are nonnegative, we may write $S_{R}^{\delta}=m_{R}^{\delta}(P)$, where $m_{R}^{\delta}(\lambda)=\zeta(\lambda / R)(1-\lambda / R)_{+}^{\delta}$ and $\zeta$ is smooth, compactly supported, and equal to 1 on $[0,1]$. Note that $\left\|\hat{m}_{R}^{\delta}\right\|_{1}=O(1)$.

Fix $f$. It suffices to show that (2) holds for $\alpha=C^{-1}$. We may assume that $f$ is supported on an arbitrarily small subset of $M$, which we can identify with a subset of $\mathbb{R}^{n}$. We then decompose $f=g+\sum_{I} b_{I}$ as before except that we use a rescaled dyadic mesh, so that the cubes have sidelength $2^{i} / R$ instead of $2^{i}$.

Since $m_{R}^{\delta}$ is bounded and supported in $[-C R, C R]$, we may dispose of the contributions of $g$ and the cubes of sidelength at most $1 / R$. Instead of a Gaussian, one uses an $S^{-\infty}$ symbol $\psi$ on $\mathbb{R}$ such that $\hat{\psi}$ is supported on $[-C, C]$ and $\psi_{R}=\psi\left(R^{-1}\right.$.) majorizes $m_{R}^{\delta}$. By Proposition 3.1(ii), the kernel of $\psi_{R}(P)$ is majorized by a constant multiple of a positive and "radial decreasing" approximation to the identity of "thickness" $1 / R$, and one can use the argument in the proof of Theorem 1.1.

The appropriate analogue of Lemma 2.1 is the following decomposition of $m_{R}^{\delta}$ :

Lemma 4.1. For each $i>0$, there exists a decomposition $m_{R}^{\delta}=m_{i}+\eta_{i} n_{i}$ such that:

(i) The functions $\hat{m}_{i}$ and $\hat{n}_{i}$ are supported on $\left[-2^{i} / R, 2^{i} / R\right]$, and each have an $L^{1}$ norm which is $O(1)$. 
(ii) For all $\lambda \in \mathbb{R}$, we have $\sum_{i=1}^{\infty}\left|\eta_{i}(\lambda)\right|^{2} \leq C$.

(iii) For a certain large $N$, we have the estimate

$$
\left|n_{i}(\lambda)\right| \leq C 2^{-\delta i}\left(1+2^{i}|1-\lambda / R|\right)^{-N} .
$$

Apart from the last part of (i), Lemma 4.1 is proved in exactly the same way as Lemma 2.1. Since $\hat{m}_{i}$ is by construction the product of $\hat{m}_{R}^{\delta}$ and a bounded expression, we see that the $L^{1}$ norm of $\hat{m}_{i}$ is $O(1)$. To prove the corresponding bound for $\hat{n}_{i}$, use (iii) to observe that $\hat{n}_{i}$ is uniformly bounded by $O\left(2^{-\delta i} 2^{-i} R\right)$, and so the $L^{1}$ norm is $O\left(2^{-\delta i}\right)=O(1)$, as desired.

The rest of the argument, deducing (2) from the above decomposition, the Cauchy-Schwarz inequality, and the restriction theorem, is virtually unchanged, apart from a rescaling by $R$. There are only two technical differences: firstly, as the restriction theorem is now discrete, one replaces a certain integral by its Riemann sum instead; and secondly, because the analogous "locality" principle (i.e. Proposition 3.2 for $p=2$ combined with Lemma 4.1(i)) is not absolute, one incurs further error terms which are $O\left(\sum_{I}|I|\right)$, but these errors are acceptable in proving $(2)$. We omit the details.

\section{ACKNOWLEDGMENT}

The author thanks E. Stein and C. Feffermann for their patience and time, and also for several observations which eventually led to substantial simplifications in this paper.

\section{REFERENCES}

1. J. Bourgain, Besicovitch-type maximal operators and applications to Fourier analysis, Geom. and Funct. Anal. 22 (1991), 147-187. MR 92g:42010

2. L. Carleson and P. Sjölin, Oscillatory integrals and a multiplier problem for the disc, Studia Math. 44 (1972): 287-299. MR 50:14052

3. M. Christ, On almost-everywhere convergence of Bochner-Riesz means in higher dimensions, Proc. Amer. Math. Soc. 95 (1985): 16-20. MR 87c:42020

4. - Weak type endpoint bounds for Bochner-Riesz multipliers, Revista Mat. Iberoamericana 3 (1987), 25-31. MR 90i:42024

5. W Weak type $(1,1)$ bounds for rough operators, Annals of Mathematics, 128 (1988): 19-42. MR 89m:42013

6. M. Christ and C. D. Sogge, The weak-type $L^{1}$ convergence of eigenfunction expansions for pseudo-differential operators, Invent. Math. 94 (1988): 421-453. MR 89j:35096

7. C. Feffermann, Inequalities for strongly singular convolution operators, Acta Math. 124 (1970), 9-36. MR 41:2468

8. — The multiplier problem for the ball, Ann. of Math. 94 (1971): 330-336. MR 45:5661

9. C. Feffermann and E. M. Stein, Some maximal inequalities, Amer. J. Math. 93 (1971): 107115. MR 44:2026

10. L. Hörmander, The spectral function of an elliptic operator, Acta Math. 121 (1968), 193-218. MR 58:29418

11. A. Seeger, Endpoint estimates for multiplier transformations on compact manifolds, Indiana Math. J. 40 (1991): 471-533. MR 92f:58166

12. _ Endpoint inequalities for Bochner-Riesz multipliers in the plane, to appear, Pacific J. Math.

13. A. Seeger and C. D. Sogge, On the boundedness of functions of (pseudo)-differential operators on compact manifolds, Duke Math. J. 59 (1989): 709-736. MR 91d:58244

14. C. D. Sogge, On the convergence of Riesz means on compact manifolds, Annals of Math. 126 (1987), 439-447. MR 89b:35126 
15. Concerning the $L^{p}$ norm of spectral clusters for second-order elliptic operators on compact manifolds, J. Funct. Anal. 77 (1988): 123-138. MR 89d:35131

16. - Fourier Integrals in Classical Analysis, Cambridge Tracts in Math. \# 105, Cambridge Univ. Press, 1993. MR 94c:35178

Department of Mathematics, Princeton University, Princeton, New Jersey 08544

E-mail address: tao@math.princeton.edu 